\title{
Rendezvous Control of Spacecraft via Constrained Optimal Control Using Generating Functions
}

\author{
By Dijian CHEN $^{1)}$ and Kenji FuJIMOTO ${ }^{2)}$ \\ ${ }^{1)}$ School of Mechanical and Electrical Engineering, China Jiliang University, Hangzhou, China \\ ${ }^{2)}$ Department of Aeronautics and Astronautics, Kyoto University, Kyoto, Japan
}

(Received June 22nd, 2017)

\begin{abstract}
This paper studies rendezvous control of spacecraft via constrained optimal control using generating functions. The problem of minimum energy control of the spacecraft transiting between specified states with constraints is formalized into the constrained optimal control problem. The generating function approach is extended to such problems by equipping with penalties. Finally, the developed technique is summarized as algorithms to successfully realize optimal rendezvous control with velocity and thrust bounds.
\end{abstract}

Key Words: Spacecraft Rendezvous, Optimal Control, Constraint, Generating Function, Penalty

\section{Nomenclature}

\begin{tabular}{|c|c|c|}
\hline$\otimes$ & $:$ & Kronecker product \\
\hline$\lambda$ & : & costate \\
\hline$\mu$ & : & penalty parameter \\
\hline$\mu_{e}$ & : & gravitational parameter of Earth \\
\hline$\omega$ & $\cdot$ & orbit rate \\
\hline$\Omega_{x}$ & : & neighborhood of origin in $\mathbb{R}^{n}$ w.r.t. $x$ \\
\hline$\Omega_{\lambda_{\mathrm{f}}}$ & : & neighborhood of origin in $\mathbb{R}^{n}$ w.r.t. $\lambda_{\mathrm{f}}$ \\
\hline$C_{k}$ & : & $k$-th component of state constraint \\
\hline$F$ & : & generating function \\
\hline $\mathcal{F}$ & : & coefficient of GF's Taylor series \\
\hline$G$ & : & universal gravitational constant \\
\hline$H$ & : & Hamiltonian \\
\hline $\mathcal{H}$ & : & expanded Hamiltonian \\
\hline$J$ & : & cost function \\
\hline$M_{e}$ & . & mass of Earth \\
\hline$P$ & : & penalty function \\
\hline $\mathcal{P}$ & . & coefficient of penalty's Taylor series \\
\hline$R_{0}$ & : & orbital radius of reference spacecraft \\
\hline - $u$ & : & input of spacecraft \\
\hline$\overline{u_{i}}, \underline{u_{i}}$ & : & upper and lower bounds of thrust \\
\hline $\mathcal{U}^{\mathrm{f}}$ & : & set of $u$ satisfying $x^{u}\left(t_{\mathrm{f}}\right)=x_{\mathrm{f}}$ \\
\hline $\mathcal{U}^{\mathrm{p}}$ & : & set of $u$ satisfying $C_{k}\left(x^{u}(t)\right) \leqslant 0$ \\
\hline $\mathcal{U}^{\mathrm{p} 0}$ & : & set of $u$ satisfying $C_{k}\left(x^{u}(t)\right)<0$ \\
\hline$v$ & : & velocity of spacecraft \\
\hline$x$ & : & state variable \\
\hline$x^{u}$ & : & solution of initial value problem \\
\hline$X$ & : & radial direction \\
\hline$\chi^{\mathrm{p} 0}$ & : & set of $x$ satisfying $C_{k}(x)<0$ \\
\hline$Y$ & : & along-track direction \\
\hline$Z$ & : & cross-track direction \\
\hline \multicolumn{3}{|l|}{ Subscripts } \\
\hline 0 & 8 & initial value of the variable \\
\hline $\mathrm{f}$ & : & terminal value of the variable \\
\hline$p$ & & variable of penalized problem \\
\hline \multicolumn{3}{|l|}{ Superscripts } \\
\hline · & . & time derivative \\
\hline * & . & optimized variable w.r.t. input \\
\hline$\wedge$ & : & variable of designed problem \\
\hline
\end{tabular}

\section{Introduction}

Many space missions, such as docking, repairing, intercepting, saving, satellite networking, large-scale structure assembling and so on, rely heavily on successful rendezvous. ${ }^{1)}$ Considering a reference spacecraft in a circular or elliptical orbit and a follower spacecraft in its neighborhood, the relative motion of the follower with respect to the reference can be described by autonomous nonlinear differential equations for which the linearized equations are known as Hill-ClohessyWiltshire (HCW) equations. ${ }^{2)}$ The problem of minimum energy control of spacecraft transiting between specified states using continuous thrust in a fixed amount of time can be formalized into a standard optimal control problem. After decades of development, much work ${ }^{3-5)}$ has been done on this topic.

Using generating functions (GFs) to evaluate the problem of optimal rendezvous control of spacecraft is a topic of interest in recent years. GF derives a family of optimal trajectories as a function of the boundary values of the states, such that it is useful in on-demand rendezvous control for different missions. Park proposes feedback control law for the spacecraft subject to the general gravity field by GFs. ${ }^{6}$ ) Bando develops a numerical technique based on Galerkin spectral method with Chebyshev polynomials ${ }^{7)}$ and also a robust controller for spacecraft rendezvous with disturbances. ${ }^{8)}$ However, both of these papers consider the unconstrained rendezvous problems. In space missions, it is necessary to consider obstacle avoidance of the spacecraft with inactive objects due to the growing amount of debris and number of spacecraft in orbit, velocity limit during transitions in order to keep the spacecraft in stable state, thrust bound and so on. All these can be regarded as constraints. The recent work by Lee presents a suboptimal continuous control algorithm that enables an active spacecraft to avoid collision with inactive space objects via GFs with penalties. ${ }^{9)}$

This paper develops a method using GF equipped with a penalty function to evaluate an energy-optimal rendezvous problem for spacecraft with constraints, particularly the velocity and thrust bounds. Section 2 reviews the HCW equation$\mathrm{s}$ and formulates the constrained energy-optimal rendezvous problem. Such a problem is formalized into a state constrained 


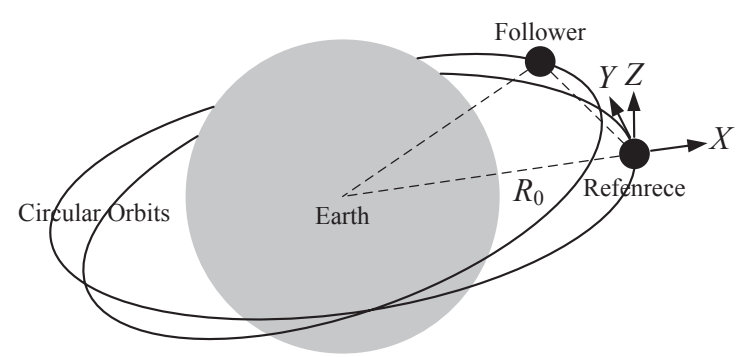

Fig. 1. Local vertical local horizontal frame.

optimal control problem and converted to an unconstrained penalized problem based on careful analyses of convexity and convergence in Section 3. Section 4 provides both the analytic and numerical solutions of the penalized problem via the GF. Two cases of velocity and thrust bounded rendezvous problems are implemented in Section 5 to illustrate the effectiveness of the proposed algorithms. Section 6 concludes the paper.

\section{Constrained Energy-Optimal Rendezvous Problem}

We review HCW equations in Section 2.1, and then formulates the constrained energy-optimal rendezvous problem in Section 2.2.

\subsection{Hill-Clohessy-Wiltshire equations}

The relative orbit between spacecrafts can be described by the famous HCW equations. ${ }^{2)}$ In this model, a so-called reference spacecraft is considered that orbits Earth in a circular trajectory (see Fig. 1). The motion of the follower spacecraft is studied from a reference frame $(X, Y, Z)$ fixed at the center of the reference spacecraft. This set of coordinate axes is called as Local Vertical Local Horizontal Frame. The relative motion in this frame is given by

$$
\begin{aligned}
& \ddot{X}=2 \omega \dot{Y}+\omega^{2}\left(R_{0}+X\right)-\frac{\mu}{R^{3}}\left(R_{0}+X\right)+u_{X}, \\
& \ddot{Y}=-2 \omega \dot{X}+\omega^{2} Y-\frac{\mu}{R^{3}} Y+u_{Y}, \\
& \ddot{Z}=-\frac{\mu}{R^{3}} Z+u_{Z},
\end{aligned}
$$

where $R=\left(\left(R_{0}+X\right)^{2}+Y^{2}+Z^{2}\right)^{1 / 2}, \omega=\left(\mu_{e} / R_{0}^{3}\right)^{1 / 2}, \mu_{e}=G M_{e}$. Note that $R_{0}$ is much larger than the relative distance between the spacecrafts. After nondimensionalization with reference length $R_{0}$ and time $1 / \omega$, and linearization about $(X, Y, Z)=$ $(0,0,0)$, we get the HCW equations as

$$
\begin{aligned}
& \ddot{X}=2 \dot{Y}+3 X+u_{X}, \\
& \ddot{Y}=-2 \dot{X}+u_{Y}, \\
& \ddot{Z}=-Z+u_{Z} .
\end{aligned}
$$

For the sake of simplicity, only the first two in-plane motions (independent of the third out-plane motion) are considered ${ }^{6}$ )

$$
\dot{x}=A x+B u,
$$

where $x:=[X, Y, \dot{X}, \dot{Y}]^{\mathrm{T}} \equiv\left[X, Y, v_{X}, v_{Y}\right]^{\mathrm{T}}, u:=\left[u_{X}, u_{Y}\right]^{\mathrm{T}}$, and

$$
A=\left[\begin{array}{cccc}
0 & 0 & 1 & 0 \\
0 & 0 & 0 & 1 \\
3 & 0 & 0 & 2 \\
0 & 0 & -2 & 0
\end{array}\right], \quad B=\left[\begin{array}{ll}
0 & 0 \\
0 & 0 \\
1 & 0 \\
0 & 1
\end{array}\right]
$$

In rendezvous problems, the initial and terminal states of the spacecraft are pre-specified as

$$
x\left(t_{0}\right)=x_{0}, \quad x\left(t_{\mathrm{f}}\right)=x_{\mathrm{f}} .
$$

\subsection{Formulation of constrained optimal control problem}

To achieve minimum energy consumption during the control of a spacecraft governed by Eq. (1) as it transits between specified states, a quadratic cost function is designed as

$$
\frac{1}{2} \int_{t_{0}}^{t_{\mathrm{f}}} u(t)^{\mathrm{T}} u(t) \mathrm{d} t .
$$

In space missions, it is necessary to consider obstacle avoidance of the spacecraft with inactive objects due to the growing amount of debris and the number of spacecrafts in orbit, and also velocity limit during transitions in order to keep the spacecraft in stable state, and so on. All these cases can be regarded as state constraints, and in general they are denoted by

$$
C_{k}(x(t)) \leqslant 0, \quad k=1,2, \cdots, s, \forall t \in\left[t_{0}, t_{\mathrm{f}}\right],
$$

where $C_{k}(\cdot): \mathbb{R}^{4} \rightarrow \mathbb{R}$ for $k=1,2, \cdots, s$.

During maneuvering, the designed thrust often can not be attained due to motor torque limit such that the real energy cost is still high, which violates the original intention of optimal control. Thus, the input constraints

$$
\overline{u_{k}} \leqslant u_{k}(t) \leqslant \underline{u_{k}}, \quad k=1,2, \forall t \in\left[t_{0}, t_{\mathrm{f}}\right],
$$

should also be taken into account.

By summarizing the above function and equations compactly, the constrained optimal control problem is formulated as

$$
\begin{aligned}
\min _{u_{\left.I_{0}, t_{\mathrm{f}}\right]}} & \frac{1}{2} \int_{t_{0}}^{t_{\mathrm{f}}} u(t)^{\mathrm{T}} u(t) \mathrm{d} t, \\
\text { s.t. } & \dot{x}=A x+B u, \quad x\left(t_{0}\right)=x_{0}, x\left(t_{\mathrm{f}}\right)=x_{\mathrm{f}}, \\
& C_{k}(x(t)) \leqslant 0, \quad k=1,2, \cdots, s, \forall t \in\left[t_{0}, t_{\mathrm{f}}\right], \\
& \overline{u_{k}} \leqslant u_{k}(t) \leqslant \underline{u_{k}}, \quad k=1,2, \forall t \in\left[t_{0}, t_{\mathrm{f}}\right] .
\end{aligned}
$$

The physical meaning of the above problem is to find an optimal thrust trajectory $u(t), t \in\left[t_{0}, t_{\mathrm{f}}\right]$, of the spacecraft governed by the dynamics (1) as it transiting between specified states $x_{0}$ and $x_{\mathrm{f}}$ in a fixed amount of time $\left[t_{0}, t_{\mathrm{f}}\right]$ satisfying the state and thrust constraints (4) and (5).

The constrained problem will be converted to an unconstrained problem by employing the penalty technique in Section 3 , and then be evaluated by the GF method in Section 4 .

\section{Problem Conversion}

The input constraint (5) can be treated as a state constraint by using a technique that will be introduced in Section 5. Then, the state-input constrained problem is reduced to the problem with state constraint only. To well present the GF method, we study the state constrained problem in a general way as follows.

\section{Problem 1.}

$$
\begin{aligned}
& \min _{u_{I_{\left.t_{0}, t_{\mathrm{f}}\right]}}} \frac{1}{2} \int_{t_{0}}^{t_{\mathrm{f}}}\left(x(t)^{\mathrm{T}} Q x(t)+u(t)^{\mathrm{T}} R u(t)\right) \mathrm{d} t, \\
& \text { s.t. } \dot{x}=A x+B u, \quad x\left(t_{0}\right)=x_{0}, x\left(t_{\mathrm{f}}\right)=x_{\mathrm{f}}, \\
& \quad C_{k}(x(t)) \leqslant 0, \quad k=1,2, \cdots, s, \forall t \in\left[t_{0}, t_{\mathrm{f}}\right],
\end{aligned}
$$


where the variables $x \in \mathbb{R}^{n}$ and $u \in \mathbb{R}^{m}$, the constants $A \in$ $\mathbb{R}^{n \times n}, B \in \mathbb{R}^{n \times m}, Q \in \mathbb{R}^{n \times n}$ is semi-positive definite, $R \in \mathbb{R}^{m \times m}$ is positive definite, $x_{0} \in \mathbb{R}^{n}$, and $x_{\mathrm{f}} \in \mathbb{R}^{n}$, and the function $C_{k}: \mathbb{R}^{n} \rightarrow \mathbb{R}, k=1,2, \cdots, s$.

This section later converts the above constrained problem to an unconstrained penalized one based on convexity and convergence analyses.

\subsection{Constrained problem and its convexity}

First, we make the following assumption.

Assumption 1. Assume the following:

(i) $C_{k}(x)$ is a convex function of $x, \forall k=1,2, \cdots, s$;

(ii) $C_{k}\left(x_{0}\right)<0$ and $C_{k}\left(x_{\mathrm{f}}\right)<0, \forall k=1,2, \cdots, s$;

(iii) $u^{*}(t)$ is continuous w.r.t. $t$;

(iv) $\mathcal{U}^{\mathrm{f}}, \mathcal{U}^{\mathrm{p} 0}$, and $\mathcal{U}^{\mathrm{f}} \cap \mathcal{U}^{\mathrm{p} 0}$ are all nonempty, where the three sets $\mathcal{U}^{\mathrm{f}}:=\left\{u \in L^{\infty}\left(\left[t_{0}, t_{\mathrm{f}}\right], \mathbb{R}^{m}\right) \mid x^{u}\left(t_{\mathrm{f}}\right)=x_{\mathrm{f}}\right\}$, $\mathcal{U}^{\mathrm{p}}:=\left\{u \in L^{\infty}\left(\left[t_{0}, t_{\mathrm{f}}\right], \mathbb{R}^{m}\right) \mid C_{k}\left(x^{u}(t)\right) \leqslant 0, k=1,2, \cdots, s, \forall t \in\right.$ $\left.\left[t_{0}, t_{\mathrm{f}}\right]\right\}$, and $\mathcal{U}^{\mathrm{p} 0}:=\left\{u \in L^{\infty}\left(\left[t_{0}, t_{\mathrm{f}}\right], \mathbb{R}^{m}\right) \mid C_{k}\left(x^{u}(t)\right)<0, k=\right.$ $\left.1,2, \cdots, s, \forall t \in\left[t_{0}, t_{\mathrm{f}}\right]\right\}$.

We know that for each input $u_{\left[t_{0}, t\right]}$, the dynamics (1) satisfying the initial condition $x\left(t_{0}\right)=x_{0}$, i.e., the initial value problem, has a unique solution $x^{u}(t)$ for $t \geqslant t_{0}$.

Next, we will show the convexity of Problem 1. To this end, we first reformulate Problem 1 as the following Problem 1'.

\section{Problem 1'.}

$\min _{\substack{u_{\left[t_{0}, t_{f}\right]} \\ u \in \mathcal{U}_{\mathrm{f}} \cap \mathcal{U}^{\mathrm{p}}}}\left(J(u):=\frac{1}{2} \int_{t_{0}}^{t_{\mathrm{f}}}\left(\left(x^{u}(t)\right)^{\mathrm{T}} Q\left(x^{u}(t)\right)+u(t)^{\mathrm{T}} R u(t)\right) \mathrm{d} t\right)$.

Then, it is easy for us to show the convexity of the set $\mathcal{U}^{\mathrm{f}} \cap \mathcal{U}^{\mathrm{p}}$ and that of the cost function $J$.

\section{Proposition 1.}

(i) Under Assumptions 1(i), 1(ii), and 1(iv), the set $\mathcal{U}^{\mathrm{f}} \cap \mathcal{U}^{\mathrm{p}}$ of Problem 1' is a convex set of the input $u$.

(ii) Under Assumption 1(iv), the cost function J(u) of Problem $l^{\prime}$ is a strongly convex function in $u, \forall u \in \mathcal{U}^{\mathrm{f}} \cap \mathcal{U}^{\mathrm{p}}$.

We omit the proof here.

Remark 1. According to Proposition 1, we know that Problem $1^{\prime}$ is the problem minimizing a strictly convex cost function of $u$ over a nonempty convex set $\mathcal{U}^{\mathrm{f}} \cap \mathcal{U}^{\mathrm{P}}$ in $u$ space, such that Problem 1' (Problem 1) has a unique global minimizer $u^{*} .^{10)}$

\subsection{Penalized problem and its convexity}

We design the following penalized problem by adding a penalty term to the cost function.

\section{Problem 2.}

$$
\begin{aligned}
& \min _{u_{\left[t_{0}, t_{\mathrm{f}}\right]}} \int_{t_{0}}^{t_{\mathrm{f}}}\left(\frac{1}{2}\left(x(t)^{\mathrm{T}} Q x(t)+u(t)^{\mathrm{T}} R u(t)\right)+\mu P(x(t))\right) \mathrm{d} t, \\
& \text { s.t. } \dot{x}(t)=A x(t)+B u(t) \\
& \quad x\left(t_{0}\right)=x_{0}, \quad x\left(t_{\mathrm{f}}\right)=x_{\mathrm{f}}
\end{aligned}
$$

where $P(x) \equiv P^{\prime}(C(x))$ and $P^{\prime}: \mathbb{R}^{s} \rightarrow \mathbb{R}$. For the penalty function, we make the following assumption.

\section{Assumption 2. Assume the following:}

(i) $P(x)$ is a convex function of $x \in \mathcal{X}^{\mathrm{p} 0}$;

(ii) $P(x) \geqslant 0, \forall x \in X^{\mathrm{p} 0}$; (iii) $P(x) \rightarrow+\infty$ when $x$ approaches the boundary of $X^{\mathrm{p} 0}$ from its interior.

Remark 2. In Problem 2 with starting and ending points ( $x_{0}$ and $x_{\mathrm{f}}$ ) in the interior, the value of the penalty grows sharply when $x$ (driven by $u$ ) approaches the boundary of the constraints such that it can prevent the state trajectory violating the constraints. This implies $u \in \mathcal{U}^{\mathrm{f}} \cap \mathcal{U}^{\mathrm{p} 0}$ in Problem 2 in fact, which is achieved by incorporating the penalty. ${ }^{11)}$ Correspondingly, the augmented cost function then can be minimized in absence of the path constraint, yielding a biased estimate of the solution of Problem 1. It is natural to imagine that we can set the factor $\mu$ small enough to reduce the bias such that the solution is close enough to that of Problem 1'. This will be investigated in the next subsection.

Now, to show the convexity of Problem 2, we reformulate it as well as follows.

\section{Problem 2'.}

$$
\begin{aligned}
& \min _{\substack{u_{\left[t_{0}, t_{\mathrm{f}}\right]} \\
u \in \mathcal{U}^{\mathrm{f}}}}\left(J_{p}(u, \mu):=\right. \\
& \left.\quad \int_{t_{0}}^{t_{\mathrm{f}}}\left(\frac{1}{2}\left(\left(x^{u}(t)\right)^{\mathrm{T}} Q\left(x^{u}(t)\right)+u(t)^{\mathrm{T}} R u(t)\right)+\mu P\left(x^{u}(t)\right)\right) \mathrm{d} t\right) .
\end{aligned}
$$

Then, convex properties of the set $\mathcal{U}^{\mathrm{f}}$ and the cost function $J_{p}$ are given in the following proposition.

\section{Proposition 2.}

(i) Under Assumption 1(iv), the set $\mathcal{U}^{\mathrm{f}}$ of Problem $2^{\prime}$ is a convex set of the input $u$.

(ii) Under Assumptions 1(i), 1(ii), 1(iv), 2(i), and 2(ii), the penalized cost function $J_{p}(u, \mu)$ of Problem $2^{\prime}$ is strictly convex in $u, \forall u \in \mathcal{U}^{\mathrm{f}}$ and $\forall \mu>0$.

We omit the proof here.

Remark 3. Proposition 2 shows that Problem $2^{\prime}$ is the problem minimizing a strictly convex cost function of $u$ over a nonempty convex set $\mathcal{U}^{\mathrm{f}}$ in $u$ space, such that Problem 2' (Problem 2) has unique global minimizer $u_{p}^{*}(\mu)$ for each specified $\mu$.

\subsection{Convergence}

We will exhibit the convergent properties of minimum cost function value and the optimal input as well as optimal state in this subsection. Before them, notice the definition of $J_{p}$ in Problem $2^{\prime}$, we can rewrite it as

$$
J_{p}(u, \mu)=J(u)+\int_{t_{0}}^{t_{\mathrm{f}}} \mu P\left(x^{u}\right) \mathrm{d} t
$$

Now first, we present the following theorem as a preparation.

Theorem $\left.1{ }^{(11,12)}\right)$. Under Assumptions 1(i), 1(ii), 1(iv), and 2, we have the following two convergent properties for the penalized cost function of Problem 2:

(i) $\lim _{\mu \rightarrow 0} J\left(u_{p}^{*}(\mu)\right)=J^{*}$;

(ii) $\lim _{\mu \rightarrow 0} \int_{t_{0}}^{t_{\mathrm{f}}} \mu P\left(x^{u_{p}^{*}(\mu)}\right) \mathrm{d} t=0$.

Theorem 1 presents the convergence of the two summands in the right hand side of Eq. (7). By combining the two conclusions of Theorem 1, we have the following corollary readily. 
Corollary 1. Under Assumptions 1(i), 1(ii), 1(iv), and 2, we have the convergence with respect to the minimum cost function values of Problems 1' and 2' as

$$
\lim _{\mu \rightarrow 0} J_{p}\left(u_{p}^{*}(\mu), \mu\right)=J^{*} .
$$

The other two main convergent properties are presented in the following theorem.

\section{Theorem 2.}

(i) Under Assumptions 1(i), 1(ii), 1(iv), and 2, we have the convergence with respect to the optimal inputs of Problems $1^{\prime}$ and $2^{\prime}$ as

$$
\lim _{\mu \rightarrow 0}\left\|u_{p}^{*}(\mu)-u^{*}\right\|_{L^{2}}=0 .
$$

(ii) Under Assumptions 1 and 2, we have the convergence with respect to the optimal states of Problems $1^{\prime}$ and $2^{\prime}$ as

$$
\lim _{\mu \rightarrow 0}\left\|x^{u_{p}^{*}(\mu)}-x^{u^{*}}\right\|_{L^{\infty}}=0 .
$$

Note that Theorem 2(i) can be proven based on Proposition 1(ii), which is an assumption in Malisani's work ${ }^{11)}$ for the nonlinear problem. We omit the proof for Theorem 2(ii) here.

In summary, by the convexity and convergence analyses in this section, we know that both Problems 1 and 2 are strictly convex problems such that they have unique global minimizers. Furthermore, the minimum cost function value as well as the optimal input and state of Problem 2 converge to the ones of Problem 1 as the factor $\mu \rightarrow 0$. Hence we can select a rather small factor $\mu$ to form a penalized problem approximating the constrained problem. From this viewpoint, we convert the constrained Problem 1 to the penalized Problem 2.

\section{Solution Using Generating Function}

This section introduces the GF approach to Problem 2. In detail, the analytic solution of Problem 2 is obtained via GF in Section 4.1. Further, since Problem 2 is a nonlinear problem, Section 4.2 gives Taylor series based implementation to provide numerical solution. It is the approximation of the solution of Problem 1. At last, the algorithms about how to solve Problem 2 numerically for different sets of boundary conditions (BCs) are summarized in Section 4.3.

\subsection{Analytic solution}

According to minimum principle, the necessary conditions for optimizing Problem 2 is

$$
\begin{aligned}
& \dot{x}=\frac{\partial H(x, \lambda)}{\partial \lambda}, \\
& \dot{\lambda}=-\frac{\partial H(x, \lambda)}{\partial x}, \\
& u_{p}^{*}=-R^{-1} B^{\mathrm{T}} \lambda,
\end{aligned}
$$

where the Hamiltonian* is defined as

$$
H(x, \lambda):=\frac{1}{2} x^{\mathrm{T}} Q x+\lambda^{\mathrm{T}} A x-\frac{1}{2} \lambda^{\mathrm{T}} G \lambda+\mu P(x),
$$

\footnotetext{
* In fact, the Hamiltonian is not only the function of $x$ and $\lambda$, but also the function of $\mu$, i.e., $H(x, \lambda, \mu)$. Since it is treated as a parameter, not a variable, for every specified $\mu$ in this section, we express the Hamiltonian as $H(x, \lambda)$ for convenience.
}

with $G=B R^{-1} B^{\mathrm{T}}$. Note that the Hamilton's equations (8) and (9) with BCs (6) form a Two Point Boundary Value Problem (TPBVP). Evaluating the optimal trajectory of Problem 2 corresponds to solving the TPBVP, which is rather difficult.

The GF, defined as $F: \mathbb{R}^{n} \times \mathbb{R}^{n} \times\left[t_{0}, t_{\mathrm{f}}\right] \rightarrow \mathbb{R}$, satisfying the Hamilton-Jacobi equation (HJE) as

$$
\frac{\partial F\left(x, \lambda_{\mathrm{f}}, t\right)}{\partial t}+H\left(x, \frac{\partial F\left(x, \lambda_{\mathrm{f}}, t\right)}{\partial x}\right)=0,
$$

specifies the family of canonical transformations $\left(x\left(t_{\mathrm{f}}\right), \lambda\left(t_{\mathrm{f}}\right)\right) \mapsto$ $(x(t), \lambda(t))$ for $t \in\left[t_{0}, t_{\mathrm{f}}\right]$ by the following relations

$$
\begin{aligned}
\lambda & =\frac{\partial F\left(x, \lambda_{\mathrm{f}}, t\right)}{\partial x}, \\
x_{\mathrm{f}} & =\frac{\partial F\left(x, \lambda_{\mathrm{f}}, t\right)}{\partial \lambda_{\mathrm{f}}} .
\end{aligned}
$$

The relations (13) and (14) also describe the time evolution of $x$ and $\lambda$ which is defined in the Hamilton's equations. Therefore, once the GF is solved from the HJE, it can be used to generate optimal trajectories $^{\dagger}$ by the evaluation of Eqs. (13) and (14) with BCs $x\left(t_{0}\right)=x_{0}$ and $x\left(t_{\mathrm{f}}\right)=x_{\mathrm{f}}$.

The following theorem gives analytic solution of Problem 2.

Theorem $3\left({ }^{13,14)}\right)$. Under Assumptions 1 and 2, the unique global minimizer of Problem 2 is

$$
u_{p}^{*}=-R^{-1} B^{\mathrm{T}} \frac{\partial F\left(x, \lambda_{\mathrm{f}}\left(x_{0}, x_{\mathrm{f}}, t_{0}\right), t\right)}{\partial x}, \quad t \in\left[t_{0}, t_{\mathrm{f}}\right],
$$

where the terminal costate $\lambda_{\mathrm{f}}\left(x_{0}, x_{\mathrm{f}}, t_{0}\right)$ is determined by solving

$$
x_{\mathrm{f}}=\left.\frac{\partial F\left(x, \lambda_{\mathrm{f}}, t\right)}{\partial \lambda_{\mathrm{f}}}\right|_{t=t_{0}} .
$$

\subsection{Numerical implementation}

Since the HJE (12) is a nonlinear partial differential equation which is difficult to find its analytic solution, we need numerical implementations to find its approximate solution. Taylor series expansion is the most popular numerical method utilized for such a purpose ${ }^{13,14)}$ so in this paper we will also use this technique.

First, we expand the nonlinear functions in Eq. (12), i.e., the GF and penalty function, as Taylor series in their arguments about zeros. To this end, we need the following assumption.

Assumption 3. For Problem 2, we assume the following:

(i) $F\left(x, \lambda_{\mathrm{f}}, t\right)$ is an analytic function of $x$ and $\lambda_{\mathrm{f}}, \forall x \in \Omega_{x}$ and $\forall \lambda_{\mathrm{f}} \in \Omega_{\lambda_{\mathrm{f}}}$;

(ii) $P(x)$ is an analytic function of $x, \forall x \in \Omega_{x}$,

(iii) $\mathcal{X}^{\mathrm{p} 0} \subseteq \Omega_{x}$.

Based on Assumption 3, we expand both $F\left(x, \lambda_{\mathrm{f}}, t\right)$ and $P(x)$ as Taylor series in their arguments about zeros as

$$
\begin{aligned}
& F\left(x, \lambda_{\mathrm{f}}, t\right)=\sum_{i=0}^{\infty} \sum_{j=0}^{i} \mathcal{F}^{(i, j)}(t) \cdot\left(x^{\otimes(i-j)} \otimes \lambda_{\mathrm{f}}^{\otimes j}\right), \\
& P(x)=\sum_{i=0}^{\infty} \mathcal{P}^{(i)} \cdot x^{\otimes i} .
\end{aligned}
$$

$\dagger$ The sufficient condition for optimality is also guaranteed. For the detail see the work of Park. ${ }^{13)}$ 
Notice Eqs. (17) and (18), the GF coefficients $\mathcal{F}^{(i, j)}(t)$ 's are undetermined, while the penalty function coefficients $\mathcal{P}^{(i)}$ 's are known.

Second, by substituting Eqs. (17) and (18) into the HJE (12), and collecting terms with same variable $x^{\otimes(i-j)} \otimes \lambda_{\mathrm{f}}^{\otimes j}$ for $j=0,1, \cdots, i$ and $i=0,1, \cdots, \infty$, we get expanded HJE. By vanishing the coefficient of each term in the expanded HJE, we get a sequence of ordinary differential equations with respect to $\mathcal{F}^{(i, j)}(t)$ 's as

$$
\dot{\mathcal{F}}^{(i, j)}(t)=-\mathcal{H}^{(i, j)}\left(\mathcal{F}^{(\cdot, \cdot)}(t), \mathcal{P}^{(\cdot)}\right),
$$

for $j=0,1, \cdots, i$ and $i=0,1, \cdots, \infty$. Furthermore, we can get the BCs of $\mathcal{F}^{(i, j)}(t)$ 's according to Eqs. (13) and (14) as

$$
\mathcal{F}^{(i, j)}\left(t_{\mathrm{f}}\right)=\left\{\begin{array}{ll}
I, & i=2, j=1 \\
0, & \text { other cases }
\end{array},\right.
$$

where $I \in \mathbb{R}^{1 \times n n}$ with all its entries equal to one. With these terminal conditions, we are able to determine all the $\mathcal{F}^{(i, j)}(t)$ 's by solving (19) in the sequence of $j=0,1, \cdots, i$ and $i=$ $0,1, \cdots, \infty$.

Once we obtain the coefficients $\mathcal{F}^{(i, j)}(t)$ 's, we obtain the GF according to Eq. (17). It enables us to obtain numerical optimal solution by the substitution of Eq. (17) into Eqs. (15) and (16).

\subsection{Algorithm}

Optimal solutions are dependent on state BCs according to Eqs. (15) and (16), while the GF coefficients are independent of state BCs according to Eq. (19). From this point of view, the GF is useful in on-demand optimal trajectory generation for the problem with different state BCs. This is the advantage of the developed method. In detail, we can divide the whole computation into two parts, between which the off-line part calculates the GF coefficients in advance, then the on-line part efficiently generates optimal solutions for different BCs. This is summarized as the following two algorithms.

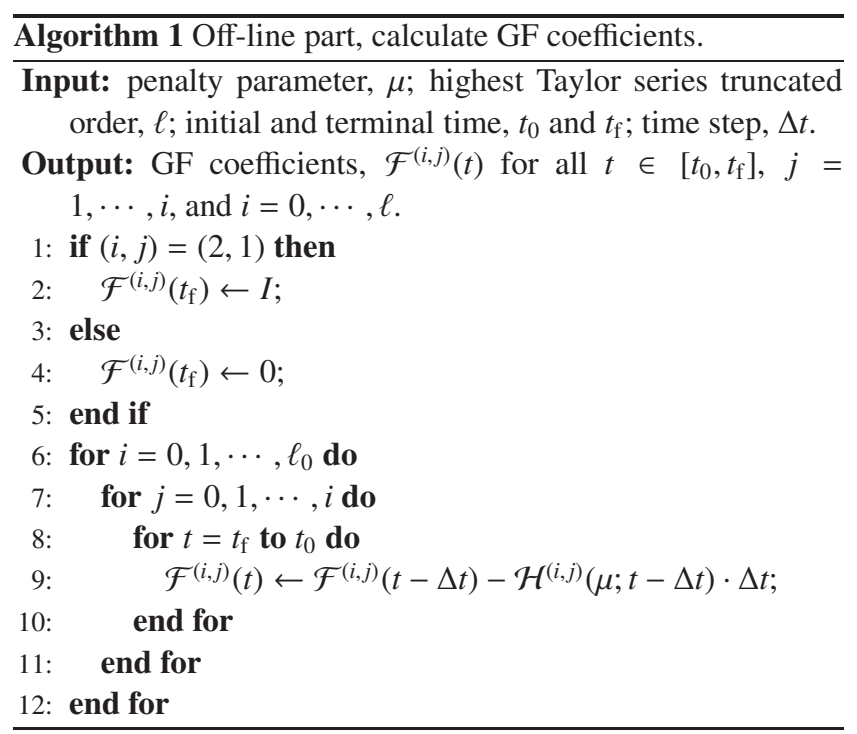

According to above algorithms, optimal solutions will be more accurate if we select greater $\ell$, i.e., truncate Taylor series up to higher orders. Since the calculation of coefficients is implemented off-line, it is free of us to choose any particular orders. From this viewpoint, though the original problem is nonlinear, we can still get solutions accurately by the developed

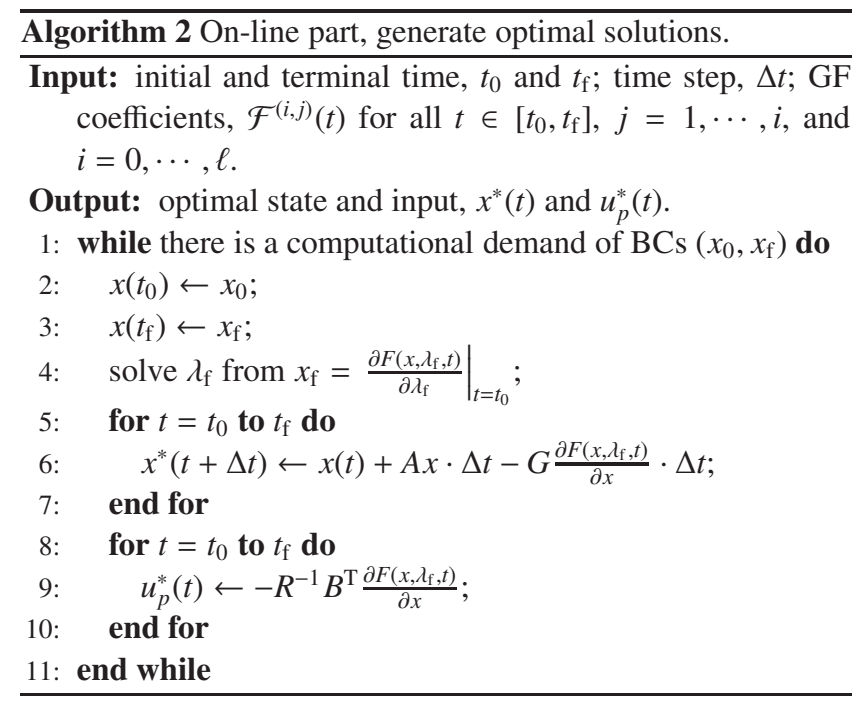

method. However, when we increase the order $\ell$, the total number of ordinary differential equations that has to be handled in off-line part also increases. For such a reason, when we select the truncated order $\ell$, both the demand of the accuracy and the computational ability of the off-line computer should be taken into account.

\section{Cases of Constrained Optimal Rendezvous}

This section applies GF method to evaluate rendezvous problems with velocity and thrust bounds in Sections 5.1 and 5.2, respectively.

\subsection{Velocity constrained optimal rendezvous}

According to Problem 1, various of constraints, such as velocity limits, position obstacles, these two mixed constraints and so on, can be well tackled by the developed GF method. In this subsection, we only consider the velocity limit example. The follower spacecraft starts from the initial positions locating along the radius 0.15 and velocities identically zero, i.e., $[0.15 \cos \theta, 0.15 \sin \theta, 0,0]^{\mathrm{T}}$ with $\theta$ varying from 0 to $2 \pi$ by the step $\pi / 8$, transits to the origin $[0,0,0,0]^{\mathrm{T}}$ in one unit time. Additionally, we also set the velocity bounds as

$$
\left|v_{X}\right| \leqslant 0.2, \quad\left|v_{Y}\right| \leqslant 0.2 .
$$

Algorithms 1 and 2 are applied to this problem. For the constraints (21), we design the penalty as

$$
\frac{1}{v_{X}+0.2}+\frac{1}{0.2-v_{X}}+\frac{1}{v_{Y}+0.2}+\frac{1}{0.2-v_{Y}},
$$

and select the penalty factor $10^{-6}$. We expand functions as Taylor series up to sixth order in off-line part. On-line part gives results shown in Fig. 2, where figures in the left column are the position and velocity trajectories for the constrained problem, while figures right column are the results in Park's work ${ }^{6}$ for the problem without velocity limits (21).

This example demonstrates the computational efficiency of the GF method for different BCs, and the comparison illustrates the effectiveness of the GF method for state constrained rendezvous control. 


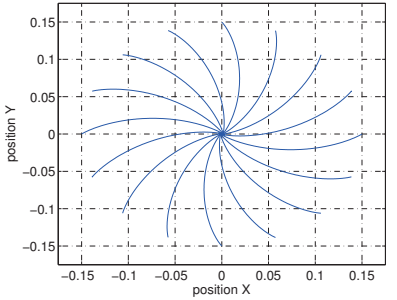

(a) Unconstrained position

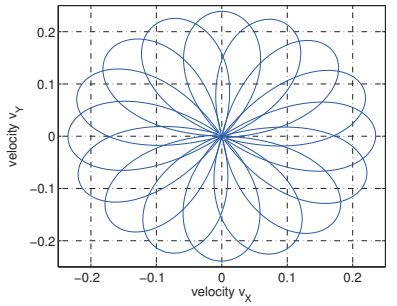

(c) Unconstrained velocity

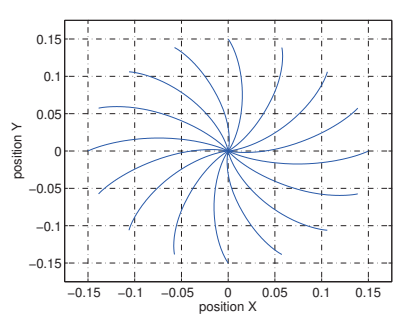

(b) Constrained position

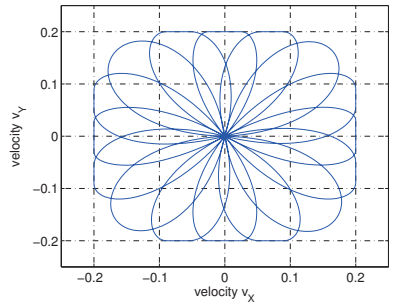

(d) Constrained velocity
Fig. 2. Comparison between unconstrained and constrained trajectories.

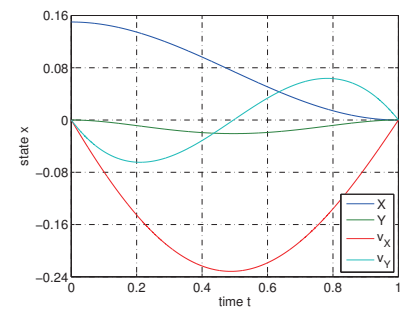

(a) State of unconstrained problem

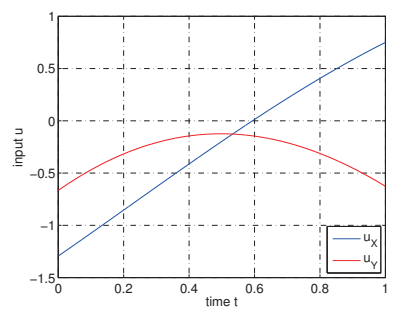

(c) Input of unconstrained problem

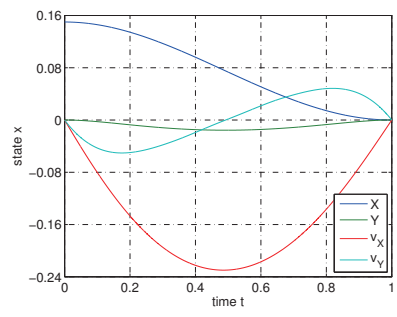

(b) State of constrained problem

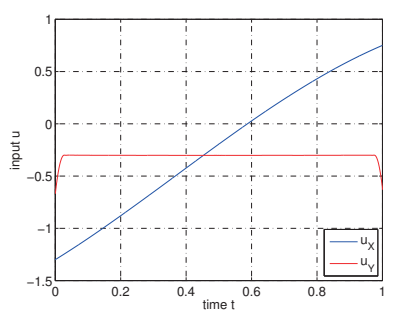

(d) Input of constrained problem
Fig. 3. Comparison between unconstrained and constrained trajectories.

\subsection{Thrust constrained optimal rendezvous}

This subsection considers the problem of spacecraft transiting from $[0.15,0,0,0]^{\mathrm{T}}$ to $[0,0,0,0]^{\mathrm{T}}$ with thrust constraints as

$$
0.3 \leqslant\left|u_{Y}(t)\right| \leqslant 0.7, \quad \forall t \in\left[t_{0}, t_{\mathrm{f}}\right]
$$

To apply the proposed algorithms, we design the problem

$$
\begin{aligned}
& \min _{\hat{u}_{\left[t_{0}, t_{\mathrm{f}}\right]}} \frac{1}{2} \int_{t_{0}}^{t_{\mathrm{f}}}\left(\hat{x}^{\mathrm{T}} \hat{Q} \hat{x}+\hat{u}^{\mathrm{T}} \hat{R} \hat{u}\right) \mathrm{d} t, \\
& \text { s.t. } \quad \dot{\hat{x}}=\hat{A} \hat{x}+\hat{B} \hat{u}, \\
& \hat{x}\left(t_{0}\right)=\hat{x}_{0}, \quad \hat{x}\left(t_{\mathrm{f}}\right)=\hat{x}_{\mathrm{f}}, \\
& 0.3 \leqslant\left|\hat{x}_{6}(t)\right| \leqslant 0.7,
\end{aligned}
$$

where $\hat{x}:=\left[x^{\mathrm{T}}, u^{\mathrm{T}}\right]^{\mathrm{T}}, \hat{x}_{0}:=\left[x_{0}^{\mathrm{T}}, u_{0}^{\mathrm{T}}\right]^{\mathrm{T}}, \hat{x}_{\mathrm{f}}:=\left[x_{\mathrm{f}}^{\mathrm{T}}, u_{\mathrm{f}}^{\mathrm{T}}\right]^{\mathrm{T}}, \hat{u}:=$ $\dot{u}, \hat{R} \in \mathbb{R}^{2 \times 2}$, and

$$
\hat{Q}:=\left[\begin{array}{cc}
0_{4 \times 4} & 0_{4 \times 2} \\
0_{2 \times 4} & R_{2 \times 2}
\end{array}\right], \hat{A}:=\left[\begin{array}{cc}
A_{4 \times 4} & B_{4 \times 2} \\
0_{2 \times 4} & 0_{2 \times 2}
\end{array}\right], \hat{B}:=\left[\begin{array}{c}
0_{4 \times 2} \\
I_{2 \times 2}
\end{array}\right] .
$$

It is obvious that a small $\hat{R}$ approximates the above designed problem to the original thrust constrained one.
Now we are able to apply Algorithms 1 and 2 to the designed problem to get approximations, where the parameter $\mu$ and truncated order $\ell$ are set as 0.01 and six. Results are shown in Fig. 3 where figures in the left column exhibit state and input trajectories of the constrained problem, while figures right column the ones for the problem without input constraint. The comparison illustrates the effectiveness of the GF method for thrust constrained rendezvous control.

\section{Conclusion}

This paper develops a method using GF equipped with penalty to solve energy-optimal rendezvous problem for spacecraft$\mathrm{s}$ with constraints, particularly the velocity and thrust bounds. Such a problem is formalized into state constrained optimal control problem and converted to unconstrained penalized problem based on careful analyses of convexity and convergence. Both analytic and numerical solutions of the penalized problem are given via the GF, and algorithms are summarized for different specified states. Two cases of velocity and thrust bounded rendezvous examples illustrate the effectiveness of the method.

\section{References}

1) Polites, M. E.: Technology of Automated Rendezvous and Capture in Space, J. of Spacecraft and Rockets, 36 (1999), pp. 280-291.

2) Clohessy, W. H.: Terminal Guidance System for Satellite Rendezvous, J. of the Aerospace Sciences, 27 (1960), pp. 653-658.

3) Marec, J.: Optimal Space Trajectories, Elsevier, New York, 1979, pp. 275-305.

4) Carter, T. E.: Optimal Power-Limited Rendezvous for Linearized Equations of Motion, J. of Guidance, Control, and Dynamics, 17 (1994), pp. 1082-1086.

5) Guelman, M. and Aleshin, M.: Optimal Bounded Low-Thrust Rendezvous with Fixed Terminal-Approach Direction, J. of Guidance, Control, and Dynamics, 24 (2001), pp. 378-385.

6) Park, C., Guibout, V., and Scheeres, D. J.: Solving Optimal Continuous Thrust Rendezvous Problems With Generating Functions, J. of Guidance, Control, and Dynamics, 29 (2006), pp. 321-331.

7) Bando, M. and Yamakawa, H.: A New Optimal Orbit Control for Two-Point Boundary-Value Problem Using Generating Functions, Advances in the Astronautical Sciences, 134 (2009), pp. 245-260.

8) Bando, M. and Yamakawa, H.: Low-Thrust Trajectory Optimization Using Second-Order Generating Functions, Proceedings of the SICE Annual Conference, Taipei, Taiwan, 2010, pp. 804-810.

9) Lee, K., Park, C., and Park, S.: Near-Optimal Continuous Control for Spacecraft Collision Avoidance Maneuvers via Generating Functions, Aerospace Science and Technology, 62 (2017), pp. 65-74.

10) Friesz, T. L.: Dynamic Optimization and Differential Games, Springer, New York, 2007, pp. 53-58.

11) Malisani, P., Chaplais, F., and Petit, N.: An Interior Penalty Method for Optimal Control Problems with State and Input Constraints of Nonlinear Systems, Optimal Control Applications and Methods, 37 (2016), pp. 3-33.

12) Lasdon, L. S., Waren, A. D., and Rice, R. K.: An Interior Penalty Method for Inequality Constrained Optimal Control Problems, IEEE Trans. on Automatic Control, 12 (1967), pp. 388-395.

13) Park, C. and Scheeres, D. J.: Determination of Optimal Feedback Terminal Controllers for General Boundary Conditions Using Generating Functions, Automatica, 42 (2006), pp. 869-875.

14) Hao, Z., Fujimoto, K., and Hayakawa, Y.: Approximate Solutions to the Hamilton-Jacobi Equations for Generating Functions: The General Cost Function Case, Proceedings of the Asian Control Conference, Istanbul, Turkey, 2013, pp. 1-6. 